\title{
TECHNICAL NOTE INTENSITY OF SHAKING GENERATED BY LARGE SHALLOW NEW ZEALAND EARTHQUAKES
}

\author{
A. L. Andrews ${ }^{1}$ and G. W. Butcher ${ }^{1}$
}

\author{
SYNOPSIS \\ There is reason to be cautious about predicting intense \\ shaking zones for large shallow New Zealand earthquakes.
}

\section{INTRODUCTION}

Spatial distributions of intensities of shaking generated by shallow earthquakes in New Zealand have been studied by Dowrick (1991). From the result of a statistical appraisal of the New Zealand record, he expressed Modified Mercalli intensity at a site in terms of earthquake magnitude and slant radius from source to site, with source represented as a point. In preparation for this work he assembled an earthquake data base, every record in which was checked for reliability - a large task involving review, data collection from abroad, and numerous reassessments to correct faultily processed information from existing files.

Then, in a further unpublished work, Dowrick et al (1992), he and his colleagues used this expression to control mapping of isoseismals. Evaluation of a team report and its intensity distribution maps suggests that the procedure works reasonably well for predicting small intensities, but that it is unreliable for high intensities which occur near the sources of large shallow focus earthquakes. Intensely shaken areas, called meizoseismal areas, are where the principal engineering and social interest is, so this unreliability is disappointing.

Specifically, when constructing a near-source picture of the distribution of MMI X shaking induced by a postulated earthquake, magnitude 7.5, effective depth (as Dowrick defined it) $7.5 \mathrm{~km}$, strike-slip source, Dowrick and his colleagues predicted the MMI X isoseismal to enclose an area c. 170 sq. $\mathrm{km}$. This area does not compare at all well with observed MMI $\mathrm{X}$ zone areas in similar New Zealand earthquakes of this century. It is not much more than $6 \%$ of Napier earthquake's c. $2700 \mathrm{sq} . \mathrm{km}$. X zone, and perhaps about the same fraction of the Murchison earthquake's X zone, the area for which has been revised downward by Dowrick, from c. $4300 \mathrm{sq}$. km. originally determined. Details have not been published.

Both these real earthquakes, though comparable, were somewhat stronger, and their sources had reverse-slip compon-

\footnotetext{
$\overline{{ }^{1} \text { Ex President and Life Member }}$
}

ents, which some investigators say induce slightly higher intensities than purely strike-slip sources do. The greater depth of Napier reduces these effects for it. The magnitude and source mechanism differences have influences much too small to account for the large disparities between observed meizoseismal zones in each of the two real earthquakes and the zone predicted for their postulated near-relative. Rational reckoning cannot close the gap.

In fact, all earthquakes listed in Dowrick's inventory as having generated shaking more intense than MMI IX (Murchison, Napier, Wairoa and Inangahua) have larger meizoseismal zones than the prediction for the postulated earthquake, despite two being smaller earthquakes, one very much smaller, and despite one of these smaller earthquakes having a greater effective depth. This is not reassuring, so it came as no surprise to find that application of the prediction method to the data base's large earthquakes, retrospective 'prediction', failed to approximate the observed meizoseismal zones. This note is concerned with possible reasons for these failures and with their correction.

\section{PRINCIPAL REASONS FOR UNRELIABILITY NEAR-SOURCE}

Among a variety of reasons, each with a potential to cause mismatch between retrospectively predicted isoseismals of high intensity shaking, made in the Dowrick way, and observed counterparts, the authors believe the following three are important, and collectively have enough effect to damage the credibility of the method, which should therefore be changed to correct them:

1. Presumption that attenuation can be assessed by supposing all energy to radiate from a point source which is located below the earthquake's epicentre.

2. Presumption that this point source is at the mid-depth of the fault or fracture surface between the rock masses which, by moving relatively, generate the earthquake.

3. The nature of the Modified Mercalli scale and the predominance of low intensity data over high intensity data in the data base. 
Also, the authors have a concern that there may have been improper use of a regression expression of intensity on radius to obtain a radius for a postulated intensity (needed for constructing an isoseismal for that intensity).

Concerning 1 and 2: Neither assumption appeals as being reasonably representative of nature. Assumption 1 ignores the fact that the slipped length of fault for an earthquake large enough to generate intense shaking is comparable to the greatest dimension of its meizoseismal zone (many tens, sometimes some hundreds, of kilometres long), and that there is a spread of sources of intense shaking along perhaps two-thirds or more of this length. In every great earthquake this is a dimension far too large to be considered to be a point. Assumption 2 is not realistic because vigorous energy will be released at a much shallower depth, and its attenuation will be overestimated by supposing travel to be from a deeper source. This objection is similar to the objection to assumption 1 .

Concerning 3: High intensity data are sparse, and are therefore overwhelmed, in regression analyses, by numerous low intensity data; but it is with high intensity shaking only that engineers and insurance people are concerned. The problem is aggravated by the character of the MMI scale, antecedents for which, devised more than a century ago, could take no account of objective measurement, because then there was no means of measuring strong earthquake motions. Evolutionary modification of the intensity scale since has not changed its subjective nature. It is, therefore, unlikely that intensity representation throughout the scale is consistent enough to justify using low intensity data to develop expressions for high intensity prediction. Until consistency of the quality that is needed has been demonstrated (for example by establishing proportionality between exp(MMI) and peak ground motion of some kind), a wise precaution would be to weight data in direct proportion to corresponding mean damage ratios, and thus, effectively, to exclude data for non-damaging intensities.

Concerning regression: A regression expression of intensity on radius (and magnitude), such as Dowrick has published and employed, has a valid use for assessing shaking a postulated earthquake on a known or suspected fault can generate at a particular site, which stands in known relation to the fault. But drawing an isoseismal is not examining intensity at a specific site. Dowrick's isoseismal plotting operation appears to use conditional mean radius as a control, and that radius should be found from a regression expression of radius on intensity. Although the two regressions, intensity on radius and radius on intensity, may use the same mathematical form, the coefficients which calibrate them will, in general, differ; and they do differ for New Zealand earthquake data when they are fitted to the form of equation which Dowrick employed.

\section{MEASUREMENT CONSIDERATIONS}

Replacement of mean horizontal radius for each isoseismal in every record in the data base, currently found from 16 radii which are measured from a central point along rays spaced at 22.5 degrees, by the measure of radius, $\mathrm{R}$, shown in Figure 1 is proposed. This would improve treatment of the attenuation path to each point on every isoseismal by making it a credible representation of a wave train path, with $R$ as that path's horizontal projection.

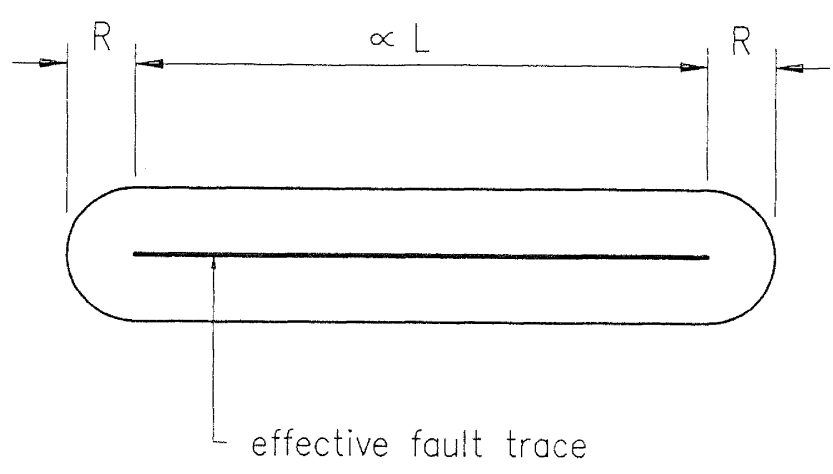

$$
\begin{aligned}
& R=\text { radius } \\
& L=\text { total slipped length of fault } \\
& \propto=\text { coefficient of effectiveness }
\end{aligned}
$$

\section{Figure 1 - An idealised isoseismal}

Causative fault slip length, $\mathrm{L}$, for an earthquake can be obtained from whichever of available expressions relating length to magnitude has strongest support from New Zealand earth scientists; and the effective length coefficient, $\alpha$, also needs their consideration. (The authors suggest that 0.67 , or thereabouts, might turn out to be appropriate - an intuitive shot that implies that end regions, each one sixth of the total length, can be ignored because there the slippage is reducing.) Then, for the data base, the measure of an isoseismal's mean horizontal radius, $\mathrm{R}$, can be obtained from the area, $\mathrm{A}$, enclosed by the isoseismal, the length, $\mathrm{L}$, and the coefficient, $\alpha$, thus:

$$
\mathrm{R}=\left(\left(\pi \mathrm{A}+\alpha^{2} \mathrm{~L}^{2}\right)^{1 / 2}-\alpha \mathrm{L}\right) / \pi
$$

The difference between a horizontal radius found this way and its corresponding value in the existing data base will be small when the ratio radius to source dimension is large. It will increase with reducing ratio to become very significant when the ratio is small enough to be characteristic of meizoseismal zones in large shallow earthquakes.

There are difficulties with mean horizontal radius as it was defined for the Dowrick study, quite apart from the obvious defect associated with it - its inability to account for extended sources that generate large earthquakes. In measuring for the data base, radius for a long, narrow shape (a typical near-source isoseismal shape for large, shallow earthquakes) cannot be determined uniquely, because the result is sensitive to orientation of the measuring template. The measuring uncertainty is exacerbated when a long narrow shape is also curved in plan.

Thus, for example, measuring to represent the inner isoseismal for the 1906 San Francisco earthquake, enclosing an arc shaped strip some $400 \mathrm{~km}$ long by generally less than $20 \mathrm{~km}$ wide (Freeman (1932)), would be impracticable by the template method. But even if it were not, a measure found (say by using a template bent to arc-matching curvature) would obviously not represent a credible attenuation path for locations on the zone boundary. And finally there is a problem of constructing a predicted isoseismal, given a mean radius, but with no measure of elongation. This has no unique solution. 
The authors recommend that slant radius be measured from the shallowest part of the slip surface that can accommodate an asperity from which vigorously energetic wave trains can emanate, rather than from the mid-depth of the fault plane. This shallowest competent part of the fault needs geotechnical consideration: provisionally it might be taken to be at the top of the middle three-quarters of the vertical projection of the fault plane. The effective depth thus defined is very much smaller than $\mathbf{h}_{\mathbf{e}}$ of the data base. Trials made with an effective depth close to this have been encouraging.

\section{OTHER CONSIDERATIONS}

Other things need attention. Among them are prospects of improving the regression data set by culling items that reason suggests should be removed, and by imposing a high magnitude cut-off. Two suggestions, and reasons for them, are:

1. Magnitude should 'saturate' for structural damage in much the way $\mathbf{M}_{\mathbf{L}}$ does for body wave amplitude and $\mathbf{M}_{\mathbf{s}}$ does for surface wave amplitude, perhaps at about $\mathbf{M}_{\text {s }}$ 7.2. Saturation is a phenomenon of vibration frequency. As magnitude increases, a growing proportion of earthquake energy is released in bands of ever reducing frequency. Higher frequency magnitude indicators have muted response to this energy, so they fail to measure the magnitude growth it represents. Saturation for structural damage (and for most other intensity indicators of the MM scale) should commence at a magnitude large enough for a significant part of the incremental earthquake energy to be generated in frequencies far below typical fundamental frequencies of structures. Whatever is agreed (by qualified people) for the saturation level, all magnitudes higher must be reduced to it for finding regression expressions and for using them. But, of course, slipped fault lengths, L, used in measuring and in constructing isoseismals, must be determined with unreduced magnitudes. There is no saturation for slipped length.

2. Small earthquake shaking is relatively much richer in high frequency energy than is large earthquake shaking, so should attenuate more with distance. Although, in near-source regions, geometric attenuation is much more important than is frequency dependent (hysteretic) attenuation, it might be worth removing earthquakes smaller than, say, magnitude 6.2 from data sets prepared for analysis, to avoid the small risk of distortions.

\section{CONCLUSION}

In its present form, the authors believe the Dowrick scheme to be unsuitable for the kinds of prediction for which it has been employed. Of special concern are failures to predict high intensity shaking zones for large shallow earthquakes in the regression expression's own data set.

Although there is no evidence to support the claim of superiority made for the method over the Smith (1978) method for predicting high intensity near-source shaking, there is evidence of good prediction for lower intensity zones, and a prospect for improvement. Moreover, Dowrick's efforts have produced a vastly improved data set, an essential for future work.
Smith's work is differently based. Only a few observations in this note, perhaps none of them, could have any relevance for an appraisal of his method; and the authors have not considered making one. Much has happened in the 14 years since Smith was published, and the work is due for revision if it is not to be abandoned; but it predicts meizoseismal zone shaking for large events more convincingly than the newer method does, so ought not be abandoned yet.

Revisions of both studies would be advantageous, if there are resources available and the work is otherwise feasible.

None of this discussion addresses the formidable problem of allowing for local (microzone) effects and nor does the method. It has been assumed that microzone induced intensity increments and decrements are balanced overall, and so cancel for damage prediction purposes - which might, or might not, be true.

\section{REFERENCES}

Dowrick, David J (1991), "A revision of attenuation relationships for Modified Mercalli intensity in New Zealand", Bull NZ National Society for Earthquake Engineering 243

Dowrick, D.J., Zhao, X.Q. and Rhoades, D.A. (1992) "Potential earthquake loss estimates for the EQC derived using different attenuation models for Modified Mercalli Intensity", unpublished report.

Freeman, John R (1932), "Earthquake damage and earthquake insurance", McGraw-Hill Book Company.

Smith, Warwick D (1978), "Spatial distribution of felt intensities for New Zealand earthquakes", NZ Jnl of Geology and Geophysics 213. 